\title{
Insulin action in the brain and how this may cause/exacerbate obesity or diabetes
}

\section{Prasad S. Dalvi}

The discovery and isolation of insulin by Banting, Best, Collip and McLeod at the University of Toronto 90 years ago was one of the greatest events in the history of medicine. Since then, insulin has become the life-saving therapy for insulindeficient type 1 diabetes patients and also patients with advanced type 2 diabetes. Presently, the incidence of type 2 diabetes has increased dramatically, from an uncommon ailment a few centuries ago to a worldwide epidemic affecting 350 million people globally. As per data published by the WHO, worldwide, $90 \%$ of people with diabetes suffer from type 2 diabetes that is developed largely due to physical inactivity and excess body weight, or obesity. Thus, obesity has become a global health issue and, as such, has been recently termed by the WHO as 'globesity'. Obesity increases mortality risks due to related complications with the main one being type 2 diabetes. ${ }^{1}$ In the fight against obesity, major efforts are currently underway to determine the central mechanisms involved in the regulation of complex processes such as appetite control and regulation of energy balance.

The main control centre of energy balance in the brain is the hypothalamus, which consists of neurons that integrate signals arriving from peripheral organs and other neurons of the nervous system to regulate appetite and maintain energy balance. ${ }^{2}$ The hypothalamic neurons are subdivided into several nuclei that control many functions such as hunger, satiety, fluid balance, and glucose regulation. Within the arcuate nucleus (ARC) of the hypothalamus, appetite-stimulating neuropeptide Y (NPY) / agoutirelated protein (AgRP) neurons promote an increase in food intake and a decrease in energy expenditure leading to the storage of energy in the form of fat. In contrast to the NPY/AgRP neurons, the pro-opimelanocortin (POMC) neurons of the hypothalamus release $\alpha-\mathrm{MSH}$, a major appetite-suppressing neuropeptide. When POMC neurons are stimulated, they promote a decrease in food intake and body weight.
Insulin is a key regulator of food intake and energy balance ${ }^{3,4,5}$ and a major metabolic hormone that regulates glucose homeostasis in the body. Insulin's role is to facilitate transportation of the food-derived glucose to target organs, such as muscle, liver or fat and make it available as an energy source. In the periphery, insulin is secreted by pancreatic beta cells. At present, there is a growing interest in insulin action on the brain in the regulation of obesity and type 2 diabetes. ${ }^{6}$ Insulin enters the brain and interacts with a number of key insulin receptor-expressing neurons in the hypothalamus that play crucial roles in the control of energy balance. ${ }^{7,8}$ In contrast to insulin's peripheral anabolic actions that increase energy storage, the central actions are shown to be catabolic that cause reduction in food intake and body weight. ${ }^{6}$ Recent research, however, suggests that there can be another side to the central insulin action, as some recent findings demonstrate that the central action of insulin may not necessarily be catabolic, but can be anabolic depending on the diet affecting peripheral insulin secretion. One study demonstrated a novel and previously unknown role for the central insulin when the peripheral insulin levels became higher than normal. When mice were exposed to a high-fat diet, the central insulin activated steroidogenic factor (SF)-1 neurons of the ventromedial nucleus in the hypothalamus and activated SF-1 neurons inhibited POMC neurons in the ARC, which resulted in suppressing the feeling of satiety and thereby increasing food consumption. ${ }^{9}$ It is quite intriguing that when the insulin receptors were deleted or inactivated in the SF-1 neurons, the mice remained lean despite consumption of high-fat diet, while their counterparts with the functional insulin receptors on the SF-1 neurons rapidly became obese. This finding suggests that increased levels of insulin during high-fat diet conditions may indirectly inhibit POMC neurons via activation of SF-1 neurons. Future research may reveal that inhibition or deletion of the insulin receptor gene and subsequent inactivation of insulin action may 
potentially provide partial protection from high-fat dietinduced hyperphagia, weight gain, and obesity.

Increased insulin levels that result from a high-fat diet have been demonstrated to decrease insulin sensitivity leading to insulin resistance in the hypothalamic neurons. ${ }^{10,11,12}$ Further, high insulin levels can develop insulin resistance in the hypothalamic neurons that could contribute to the development of obesity. ${ }^{13}$ (In this context, it must be noted that the hypothalamic obesity occurs due to insulin resistance caused by hyperinsulinemia). ${ }^{14}$ In healthy humans, the consumption of a regular diet stimulates insulin to be released from the pancreas within the normal range. In contrast, a chronic high-fat diet causes excessive insulin release from the pancreas, exposing brain structures to high levels of insulin, and thereby causing insulin resistance in some neurons or over-activation of insulin receptors in other neurons. Both actions can disrupt insulin-mediated regulation of appetite and energy balance that may result in excessive food intake, surplus energy storage and obesity. This effect of exposure of hypothalamic regions to high levels of insulin could constitute an effective evolutionary adaptation by the brain to an irregular food supply and extended periods of hunger. During a period where an excess of food is available, the excessive insulin release could potentially lead to an increase in appetite and a reduction in calorie expenditure through temporary insulin resistance in appetite-suppressing neurons, such as POMC neurons, and overstimulation in neurons, such as SF-1neurons, that further inhibit POMC neurons. The end result was a surplus of energy reserves and weight gain that were necessary to sustain life in the periods of food deprivation. In the early human era, this evolutionarilyconserved mechanism was turned "on" and "off" depending on the availability of the food to properly regulate appetite and energy balance. However, it seems that during the present period of abundance in the developed world, this mechanism is constantly turned "on" leading to an increase in appetite and fat storage.

Overall, normal insulin actions in the brain are essential in the regulation of energy balance; however, excessive insulin exposure is harmful, as it may lead to obesity and further to type 2 diabetes. Thus, if a well-balanced and healthy diet is followed, the resulting normal levels of insulin are beneficial, but as soon as the insulin levels become excessive and chronically remain pathological, the brain triggers reserved mechanisms, such as inhibition of appetite-suppressing neurons, resulting in surplus of energy intake and storage that may ultimately lead to obesity or diabetes.

\section{References}

1. Freedman DM, Ron E, Ballard-Barbash R, Doody MM, Linet MS. Body mass index and all-cause mortality in a nationwide US cohort. Int J Obes (Lond) 2006;30(5):822-9.

2. Elmquist JK, Marcus JN. Rethinking the central causes of diabetes. Nat Med 2003;9(6):645-7.

3. Ahima RS, Flier JS. Leptin. Annu Rev Physiol 2000;62:413-37.

4. Halaas JL, Gajiwala KS, Maffei M, Cohen SL, Chait BT, Rabinowitz D, Lallone RL, Burley SK, Friedman JM. Weight-reducing effects of the plasma protein encoded by the obese gene. Science 1995;269(5223):543-6.

5. Bagdade JD, Bierman EL, and Porte Jr D. The significance of basal insulin levels in the evaluation of the insulin response to glucose in diabetic and nondiabetic subjects. J Clin Invest 1967;46(10):1549-57.

6. Niswender KD, Baskin DG, Schwartz MW. Insulin and its evolving partnership with leptin in the hypothalamic control of energy homeostasis. Trends Endocrinol Metab 2004;15(8):362-9.

7. Corp ES, Woods SC, Porte Jr D, Dorsa DM, Figlewicz DP, Baskin DG. Localization of $125 \mathrm{I}$-insulin binding sites in the rat hypothalamus by quantitative autoradiography. Neurosci Lett 1986;70(1):17-22.

8. Marks JL, Porte Jr D, Stahl WL, Baskin DG. Localization of insulin receptor mRNA in rat brain by in situ hybridization. Endocrinology 1990;127(6):32346.

9. Klöckener T, Hess S, Belgardt BF, Paeger L, Verhagen LAW, Husch A, Sohn J-W, Hampel B, Dhillon H, Zigman JM, Lowell BB, Williams KW, Elmquist JK, Horvath TL, Kloppenburg P, Brüning JC. High-fat feeding promotes obesity via insulin receptor/PI3K-dependent inhibition of SF-1 VMH neurons. Nat Neurosci 2011;14(7):911-8

10. De Souza CT, Araujo EP, Bordin S, Ashimine R, Zollner RL, Boschero AC, Saad MJA, Velloso LA. Consumption of a fat-rich diet activates a proinflammatory response and induces insulin resistance in the hypothalamus. Endocrinology 2005;146(10):4192-9.

11. Benoit SC, Kemp CJ, Elias CF, Abplanalp W, Herman JP, Migrenne S, Lefevre A-L, Cruciani-Guglielmacci C, Magnan C, Yu F, Niswender K, Irani BG, Holland WL, Clegg DJ. Palmitic acid mediates hypothalamic insulin resistance by altering PKC-theta subcellular localization in rodents. J Clin Invest 2009;119(9):2577-89.

12. Mayer CM, Belsham DD. Palmitate attenuates insulin signaling and induces endoplasmic reticulum stress and apoptosis in hypothalamic neurons: rescue of resistance and apoptosis through adenosine 5 ' monophosphateactivated protein kinase activation. Endocrinology 2010;151(2):576-85.

13. Mayer CM, Belsham DD. Central insulin signaling is attenuated by long-term insulin exposure via insulin receptor substrate-1 serine phosphorylation, proteasomal degradation, and lysosomal insulin receptor degradation. Endocrinology 2010;151(1):75-84.

14. Shanik MH, Xu Y, A krha J, Dankner R, Zick Y, Roth J. Insulin resistance and hyperinsulinemia. Diabetes Care 2008;31(Supplement 2):S262-S268. 


\section{Comments on Dialogue Piece (from HSI Members)}

\section{Comment 1: On the role of brain insulin action for obesity and diabetes}

This article is outlining the relationship between a high fat diet, resulting insulin action in the brain and the development of diet related chronic disease including obesity and diabetes. The author begins by describing the shocking statistics that reflect present day burden of obesity and highlights the World Health Organizations (WHO) recent use of the term 'globesity' to emphasize the global nature of the obesity epidemic. In fact obesity is not the only health related outcome claiming 'epidemic' status. Besides the global epidemic of obesity, the WHO also refers to the epidemic of diabetes, cardiovascular disease (i.e. heart attack and stroke) and cancer. ${ }^{1}$

In response to these epidemics, the author suggests that, 'major efforts are underway to determine the central mechanisms involved in the regulation of complex processes such as appetite control and energy homeostasis'. This is an important dimension of understanding obesity and diabetes, however one could argue for other dimensions of the aetiology of chronic disease includes broader factors acting upon these aspects of human physiology. For example, the WHO also makes reference to the 'obesogenic environment', which is a term to describe the many dimensions of our 'modern environment' that encourage consumption and discourage expenditure. ${ }^{2}$ It is characterized by plentiful access to cheap, energy dense, nutritionally deficient foods that appeal to our pocket books and taste preference for salty/fatty foods and our countless technological conveniences including cars, elevators and escalators to save us the trouble of expending precious calories. $^{3}$

Although the environment was not discussed directly, the author acknowledged that our evolved physiological mechanisms for dealing with energy imbalance remains programmed for a world of scarcity and constant physical work. In my view this is a profound statement; one that leaves those of us interested in how we reverse these global epidemics, vigorously scratching our heads. From the perspective of human physiology within an evolutionary context, as described in this article, it seems that there are a few possible paths forward. One is to fundamentally change human physiology (i.e. insulin actions in the brain) to favour an environment of abundance; or begin system level intervention to do the necessary political, economic, social and cultural work needed to create more balance in our modern environments so our physiology is not overwhelmed by excess fatty diets, increased insulin in the body and resulting obesity and diabetes. There is, of course, one additional pathway. However, it is the pathway we have been stumbling down for the past three decades, the one that has left those of us in disease prevention frustrated and with ever increasing prevalence of global chronic disease. I am referring to the behavioural path. The author of this article alludes to it when the author states, 'To control the detrimental effects of excessive insulin the new mantra should be: low-fat food, less brain insulin, lean body, and longer healthy life.' I would argue that this mantra has been given serious scientific exploration and testing over the past few decades, with modest and mixed results. ${ }^{4}$ It is often called the Eat-Less-Move-More (ELMM) mantra and exclusively appeals to an individual's behaviour while simultaneously neglecting their physiology and the environment in which that physiology evolved. In practice a focus on isolated health behaviour can often result in not only failure to make behavioural changes but stigmatization of the individual seen as responsible for their own disease status. $^{5}$

As a scientific community, we need research that explores the physiological processes as in this article. However, we must also be mindful of applying a behavioural solution to problems of primarily physiological and environmental origins.

\section{Tarra L. Penney (HSI Senior Editor)}

Tarra L. Penney is a research associate and graduate student in the area of chronic disease prevention and population health intervention research at Dalhousie University.

\section{References}

1. WHO | The Atlas of Heart Disease and Stroke [Internet]. WHO. [cited 2012 Apr 8]. Available from: http://www.who.int/cardiovascular_diseases/ resources/atlas/en/

2. WHO | Controlling the global obesity epidemic [Internet]. WHO. [cited 2012 Apr 8]. Available from: http://www.who.int/nutrition/topics/obesity/en/

3. Kirk SFL, Penney TL, McHugh T -L. F. Characterizing the obesogenic environment: the state of the evidence with directions for future research. Obesity Reviews. 2010 Feb 1;11(2):109-17.

4. Kirk SFL, Penney TL, McHugh T-L, Sharma AM. Effective weight management practice: a review of the lifestyle intervention evidence. International Journal of Obesity. 2011 Apr 12;36(2):178-85.

5. Puhl RM, Heuer CA. The Stigma of Obesity: A Review and Update. Obesity. 2009 Jan 22;17(5):941-64. 


\section{Comments on Dialogue Piece (from HSI Members)}

\section{Comment 2: On the role of brain insulin action for obesity and diabetes}

While I agree with the overall sentiment of Dr. Dalvi that obesity can lead to impaired insulin signaling in the brain, he only touches on the positive role that insulin plays in maintenance of a lean body composition. Before I expand on insulin's role as a satiety signal and vital glucostatic regulator, I would like to highlight its evolutionary importance.

Insulin has been shown to play an important role in regulating life span, growth, and reproduction, as well as energy homeostasis in organisms as primitive as Caenorhabitis elegans (roundworm) and Drosophila melanogaster (fruitfly). ${ }^{1,2,3}$ Furthermore, it has been shown that humans share the same conserved molecular mechanism of insulin signaling used by these simple organisms. ${ }^{4}$ With that said, what positive roles does insulin have within the brain that have allowed it to stay relatively unchanged throughout many organisms and years of natural selection. Firstly, as mentioned in Dr. Dalvi's piece, insulin can act as a satiety signal, making individuals feel full after eating a meal. It is hypothesized that insulin's direct induction of the appetite supressing $\alpha$-Melanocyte-stimulating hormone $(\alpha-\mathrm{MSH})$ release from POMC neurons and suppression of the orexogenic neuropeptide $Y$ (NPY) release from NPY neurons causes suppression of feeding behavior. ${ }^{5,6}$ Insulin has been shown to regulate glucose homeostasis centrally by altering hepatic glucose production (HGP). Infusion of insulin to the brain has been shown to substantially decrease hepatic glucose output in mice. ${ }^{7}$ Furthermore, in mice lacking the insulin receptor (IR) within the feeding-related agouti-related protein (AgRP) neurons in the hypothalamus, the effect of central administration of insulin is attenuated. ${ }^{8}$ In whole body IR knockout mice that have IR restored specifically within AgRP neurons, infusion of insulin once again suppresses HGP. ${ }^{9}$ This finding suggests that not only does insulin play an important role in regulation of glucose within the periphery, but can exert effects within the brain to regulate peripheral tissue glucose utilization.

In 2010, Paranjape et al. proposed another mechanism by which insulin regulates peripheral tissue glucose utilization, this time by regulating release of glucagon (the hormone responsible for decreasing glucose uptake in the periphery). This group found that glucose acts centrally within a subset of ventromedial hypothalamic neurons to regulate glucagon secretion within the pancreas in both hypoglycemic and normal conditions. ${ }^{10}$ Taken together, these findings suggest that the role of insulin in the brain is to fine-tune the ability of peripheral tissues to uptake glucose, thereby increasing the energy available to these neurons. By decreasing hepatic glucose production, insulin increases energy stores in the form of glycogen to be used in times of metabolic stress. The decrease in glucagon secretion further decreases the amount of glucose expelled from peripheral tissues into the blood, further increasing total body glucose uptake. In a normal individual, following a meal, insulin secreted by the pancreas is sensed within the brain. Signals are then sent which modify feeding behavior via NPY/POMC thereby decreasing the craving to eat.

The evolutionarily conserved mechanism of insulin action is in place to keep our bodies healthy and lean, and only after severe damage or chronic abuse from sugary foods and drinks does this highly ordered system malfunction. It is important to acknowledge everything that insulin does to keep our body in balance. Collectively, the vital role that insulin plays centrally in energy balance and homeostasis has made it an evolutionarily conserved component of our homeostatic system.

\section{Sean A. McFadden (HSI Managing Editor)}

Sean A. McFadden is a Master's graduate student in the area of diabetes research and neuroendocrinology at the University of Toronto.

\section{References}

6. Rulifson, E.J., Kim, S.K., and Nusse, R. 2002. Ablation of insulin-producing neurons in flies: growth and diabetic phenotypes. Science. 296:1118-1120.

7. Wolkow, C.A., Kimura, K.D., Lee, M.S., and Ruvkun, G. 2000. Regulation of C. elegans life-span by insulinlike signaling in the nervous system. Science. 290:147-150.

8. Tissenbaum, H.A., and Ruvkun, G. 1998. An insulin-like signaling pathway affects both longevity and reproduction in Caenorhabditis elegans. Genetics. 148:703-717.

9. Cheng, C.L., Gao, T.Q., Wang, Z., and Li, D.D. 2005. Role of insulin/insulinlike growth factor 1 signaling pathway in longevity. World J. Gastroenterol. 11:1891-1895.

10. Benoit SC, Air EL, Coolen LM, Strauss R, Jackman A, Clegg DJ, Seeley RJ, Woods SC. The catabolic action of insulin in the brain is mediated by melanocortins. J. Neurosci. 2002;22:9048-9052.

11. M W Schwartz, A J Sipols, J L Marks, G Sanacora, J D White, A Scheurink, S E Kahn, D G Baskin, S C Woods and D P Figlewicz. Inhibition of hypothalamic neuropeptide Y gene expression by insulin. Endocrinology. 1992;130:36083616.

12. Obici S, Zhang BB, Karkanias G, Rossetti L. Hypothalamic insulin signaling is required for inhibition of glucose production. Nat Med. 2002;8(12):13761382.

13. Könner $A C$, Janoschek R, Plum L, Jordan SD, Rother E, Ma X, Xu C, Enriori $P$, Hampel B, Barsh GS, Kahn CR, Cowley MA, Ashcroft FM, Brüning JC. Insulin action in AgRP-expressing neurons is required for suppression of hepatic glucose production. Cell Metab. 2007;5(6):438-449.

14. Lin $H V$, Plum L, Ono $H$, Gutiérrez-Juárez $R$, Shanabrough $M$, Borok $E$, Horvath TL, Rossetti L, Accili D. Divergent regulation of energy expenditure and hepatic glucose production by insulin receptor in agouti-related protein and POMC neurons. Diabetes. 2010;59(2):337-346.

15. Paranjape SA, Chan O, Zhu W, Horblitt AM, McNay EC, Cresswell JA, Bogan $\mathrm{JS}$, McCrimmon RJ, Sherwin RS. Influence of insulin in the ventromedial hypothalamus on pancreatic glucagon secretion in vivo. Diabetes 2010. 59: 1521-152 


\section{Author's Response to Comments}

I would like to thank Mr. Sean Mcfadden and Ms. Tarra L. Penney for their thoughtful responses to my article and for raising a number of valid points. I will address the responses of each in sequence and clarify my points of view further.

Mr. Mcfadden notes quite rightly that I did not address many beneficial insulin actions that are mediated via central nervous system to regulate peripheral glucose levels. Rather, I presented the relatively unknown actions of the excessive insulin in the brain that may contribute to development of obesity. I particularly focused on that aspect, because it is the disruption of the normal insulin signaling that leads to type 2 diabetes.

It is now known that the insulin and insulin signaling pathways are evolutionarily well conserved among multicellular organisms, including both vertebrates and invertebrates. ${ }^{1}$ Apart from insulin's role as a major regulator of intracellular and blood glucose levels, it plays a pivotal role in cellular developmental processes such as growth and aging. ${ }^{1}$ Although all these functions are evolutionarily very well conserved across almost all species, unlike humans, most multicellular organisms are infrequently exposed to high sugar and therefore high insulin levels. Thus, these organisms may never suffer from the detrimental effects of pathological levels of insulin. Recently, it was found that altered insulin signaling resulting from brain insulin resistance may play a major role in the pathogenesis of neurodegenerative diseases, such as Alzheimer's disease. ${ }^{2}$ There are several factors, such as toxic lipids, that may cause impairment in brain insulin signaling and lead to neuronal death. Therefore, it is tempting to speculate that increased brain insulin levels also contribute to neurodegeneration via insulin resistance.

In agreement with Mr. Mcfadden's response, the satiety action of brain insulin must not be ignored. Investigations have shown that insulin and a fat cell-derived satiety hormone leptin send signals, known as adiposity signals, to the brain about peripheral energy status. However, the signaling function of these hormones remains intact until their respective levels are within physiological limits. Once the levels become supra-physiological or pathological, neurons develop resistance to both hormones and the brain stops receiving the signals about the energy stores in the body. The absence of adiposity signals may cause the brain to perceive that there is a constant energy deficit that further causes the brain to trigger appetite-stimulating
NPY/AgRP neurons to induce excess energy intake and surplus fat storage. This can be exemplified by increased appetite and fat mass in obese individuals, most of whom have leptin and insulin resistance. As insulin resistance is developed in humans who consume high-glucose or highfat diet, it is no wonder why wild animals remain immune to obesity and therefore insulin resistance and all deleterious effects associated with it.

Mr. Mcfadden mentions about the role of brain insulin in glucose regulation by its action on liver. There is no doubt that insulin and glucagon are potent regulators of glucose metabolism mainly acting on liver. Although both exert counter-regulatory actions on each other, during embryonic stage, the cells that generate these hormones arise from a common precursor cell. ${ }^{3,4}$ This suggests that evolutionarily these hormones are closely related. It is the glucagon that is 100 -fold active than insulin during embryogenesis. ${ }^{3}$ This indicates that glucagon, the hormone that increases glucose levels in the blood, predominates over insulin at the initial development of an embryo. But as the embryo grows and gradually the glucose levels increase in the organism, insulin levels start rising to regulate the glucose utilization and counter-regulate glucagon action. Insulin's direct action on muscle and liver, and its indirect regulation via brain to increase glucose uptake by peripheral organs are necessary to prevent harmful effects of high glucose levels. However, with the development of peripheral and brain insulin resistance, insulin's counter-regulatory action on glucagon is impaired, leading to a marked increase in liver glucose output and break-down of fat to increase fatty acid levels. Eventually, this may lead to metabolic crisis such as diabetic coma. Thus, for healthy life it is imperative to maintain optimal insulin levels at all times.

Ms. Tarra L. Penney reminds us that the world is facing not only obesity epidemic, but also epidemics of several other diseases, such as cardiovascular diseases and cancer. Unfortunately, gathered epidemiological data suggest that a major contributor of all these diseases and metabolic disorders is the excessive fat mass and obesity. Indeed, I agree with Ms. Penney that we live in 'obesogenic environment' in the developed world. Absolutely, it is hard to make healthy choices among multiple unhealthy lifestyle choices that surround us in the "modern Western environment". The evolutionarily-conserved mechanisms that were triggered rarely or intermittently are constantly turned on and are reset to cope with this environment of 


\section{Author's Response: Insulin action in the brain...}

abundance in such a way that is not beneficial to human health. For example, recently, it was found that total sperm count was significantly negatively correlated with body mass index in young adults and sperm quality was compromised in obese individuals. ${ }^{5,6}$

Ms. Penney suggests two ways to deal with these global epidemics. As mentioned in her response, one way is to fundamentally change human physiology rather than to change an environment of abundance. At present, pharmacological manipulations to prevent brain insulin resistance in humans are far from our reach. As mentioned earlier, insulin is evolutionarily well conserved, and I wonder about its function and overall fate over the next hundreds or thousands of years, if human brains continue to be exposed to high insulin levels.

Ms. Penney further points out the failures of the Eat-LessMove-More (ELMM) mantra in the combat against obesity and diabetes. As obesity is a complex issue involving both physiological and environmental factors, she recommends focusing on the broader social and enviornmental factors. Although this option may demand a lot of resources at a population health level, it is quite feasible if strong government and public initiative support are sought. Finally, I feel that only proper education, counselling based on scientific findings, and constant motivation to fight against obesity will bring some hope.

\section{References}

1. Brogiolo, W., et al., An evolutionarily conserved function of the Drosophila insulin receptor and insulin-like peptides in growth control. Curr Biol, 2001. 11(4): p. 213-21.

2. de la Monte, S.M., Insulin resistance and Alzheimer's disease. BMB Rep, 2009. 42(8): p. 475-81.

3. Rall, L.B., et al., Early differentiation of glucagon-producing cells in embryonic pancreas: a possible developmental role for glucagon. Proc Natl Acad Sci U S A, 1973. 70(12): p. 3478-82.

4. Herrera, P.L., Adult insulin- and glucagon-producing cells differentiate from two independent cell lineages. Development, 2000. 127(11): p. 2317-22.

5. Paasch, U., et al., Obesity and age affect male fertility potential. Fertil Steril, 2010. 94(7): p. 2898-901.

6. Fariello, R.M., et al., Association between obesity and alteration of sperm DNA integrity and mitochondrial activity. BJU International, 2012: p. no-no.

Prasad S. Dalvi

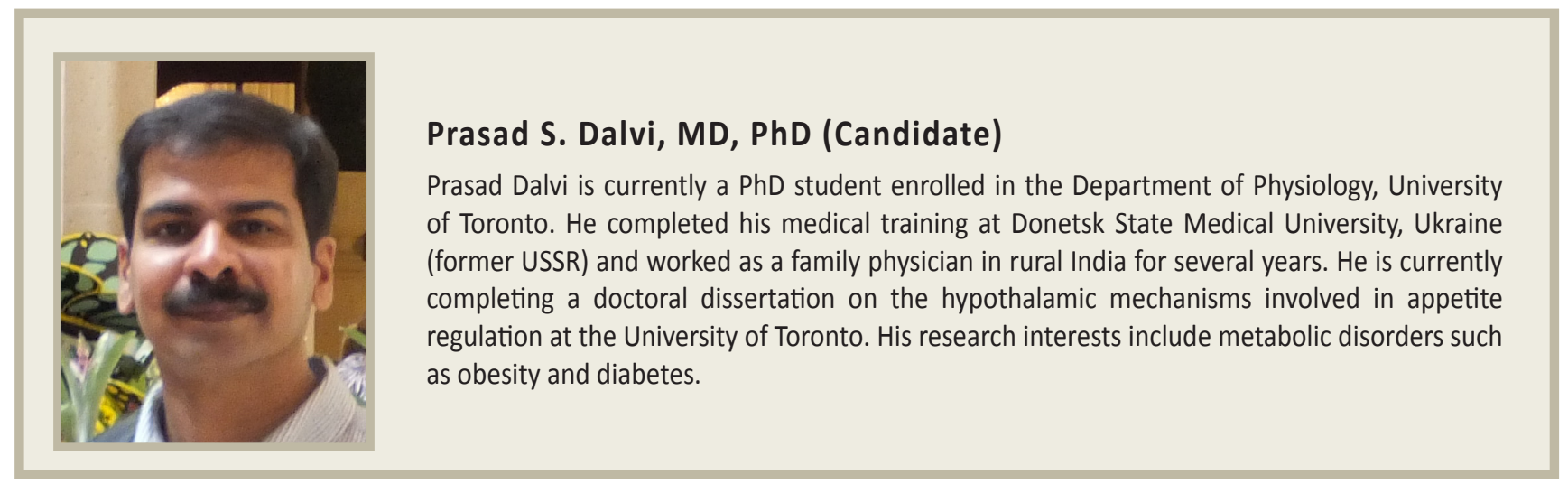

